\title{
Pathology of experimentally re-innervated skeletal muscle
}

\author{
VICTOR DUBOWITZ ${ }^{1}$ \\ From the Department of Cell Biology, Institute for Muscle Disease, \\ New York 21, New York, U.S.A.
}

Structural abnormalities such as ringbinden (striated annulets) and schlangelungen (snake coils) were already recognized by the early German histologists, but their significance is still debated. Heidenhain (1918) and Wohlfart (1951) noted their high incidence in dystrophia myotonica. They are, however, occasionally found in muscular dystrophy and other muscular disorders and even in normal muscle, particularly in the elderly (Wohlfart, 1932, 1951). Perry, Smith, and Wrenn (1956) found ringbinden in the histologically normal muscle of a youth with spasticity, and subsequently found three further examples in a review of 58 previous muscle biopsies. Most authors (Bergstrand, 1938; Wohlfart, 1951; Günther, 1952; Perry et al., 1956) have looked upon ringbinden as pathological muscle fibres, but others (Adams, Denny-Brown, and Pearson, 1962) regard them as an artifact, possibly of fixation.

Recently Engel (1961) observed 'target' fibres in 27 of 46 human biopsies with classical features of denervation and concluded that they are a sign of denervation. All these target fibres were of normal or reduced size. He subsequently differentiated three-zone target from two-zone targetoid fibres (Engel, 1962). Target and targetoid fibres bear some resemblance to the core fibres of central core disease (Dubowitz and Pearse, 1960; Engel, Foster, Hughes, Huxley, and Mahler, 1961). Hogenhuis and Engel (1965) found no target fibres in guinea-pig muscle during a period of 11 weeks after denervation, but observed coil fibres after seven weeks. This corresponded to the time at which hypertrophy of some fibres also first became evident.

In contrast to the extensive literature on the effects of denervation on the muscle, there has been practically no interest in the changes during re-innervation. We have recently had the opportunity of making some histochemical and histological observations on muscle during re-innervation, in the course of our studies on the effects of cross-innervation on fast and slow skeletal muscle.

1Present address: Department of Child Health, University of Sheffield, Sheffield 10, England
In a fascinating series of experiments Buller, Eccles, and Eccles (1960b) demonstrated that crossunion of the nerves to the fast flexor hallucis longus and the slow soleus muscles in the cat resulted in a slowing of contraction time in the flexor hallucis longus and an acceleration in the soleus. This effect was abolished if, in addition, the spinal cord were 'isolated' from all incoming impulses by transection and deafferentiation. They concluded that the contractile properties of skeletal muscle are under a neural influence from the central nervous system and postulated a chemical substance passing down the axon, traversing the neuromuscular junction and spreading throughout the muscle fibre.

A study has been made of the effects of crossunion of the nerves to fast and slow muscle on the structural and histochemical properties of the muscle and their correlation with the physiological changes. During the phase of re-innervation of the muscle, both after cross-union and self-union of the sectioned motor nerves, there was a strikingly high incidence of abnormal muscle fibres. These included giant fibres of apparently normal structure, coil fibres and ringbinden, target and targetoid fibres, and various alterations in the enzyme histochemistry of the fibres. In addition a striking transformation occurred in the whole enzyme pattern of the muscle after cross-innervation.

It thus appears that the central nervous system may have a very marked influence not only on the contractile properties of the muscle, but also on the structural and metabolic character. Abnormal forms of muscle fibre probably represent an abnormal morphogenesis during the process of re-innervation of muscle fibres.

\section{MATERIALS AND METHODS}

OPERATIVE PROCEDURES Three series of animals were studied, namely, adult cats, newborn kittens, and newborn rabbits. The animals were anaesthetized with pentobarbitone sodium (50 mg./kg. intraperitoneally), or a mixture of air and ether, which was found safer in the newborn animals. 
Cross-union of the nerves to the soleus and flexor hallucis longus or flexor digitorum longus was effected with human hair under full aseptic precautions. In a control series self-union of these nerves was done by section and re-approximation. All nerve sutures were made as close as possible to the insertion of the nerve into the muscle. Denervation was performed in a number of cases by removing a segment (usually about 3 to $5 \mathrm{~mm}$.) of the nerve. In a fourth group tenotomy of the distal end of these individual muscles was performed between nylon ligatures. A number of unoperated limbs were studied as controls. Details of the animals surviving beyond the first postoperative week are included in Tables I to V. One litter of eight newborn rabbits died of inanition six to seven days postoperatively, presumably as a result of maternal rejection. The muscles of this litter and also a number of other animals surviving for short periods were studied for the effects of denervation.

ACUTE EXPERIMENTS After periods ranging from 26 to 33 weeks in the cats, 18 to 20 weeks in the kittens, and five to 14 weeks in the rabbits, the animals were anaesthetized with pentobarbitone sodium, $50 \mathrm{mg}$. $/ \mathrm{kg}$. intraperitoneally. The contractile properties of the soleus, FHL, and FDL were measured on a Statham G1-4-250 strain gauge and Sanborn 150 recorder, with care being taken to preserve the blood supply of the muscles during this procedure. The muscles were then dissected out and weighed. Three specimens, from the proximal, middle and distal thirds of each muscle, were then prepared, mounted in a transverse plane on a slice of cork, and rapidly frozen in liquid nitrogen for histochemical and histological study (Dubowitz and Pearse, 1964). Sections were cut at $5 \mu$ in a cryostat $\left(-30^{\circ} \mathrm{C}\right.$.) and assessed for activity of phorphorylase (Takeuchi and Kuriaki, 1955; Eränkö and Palkama, 1961; Sawyer, Sie, and Fishman, 1965), reduced nicotinamide adenine dinucleotide $\left(\mathrm{NADH}_{2}\right)$ diaphorase (Scarpelli, Hess, and Pearse, 1958), and myosin adenosine triphosphatase (ATPase) (Padykula and Herman, 1955). In addition the modified Gomori trichrome stain was done (Engel and Cunning ham, 1963).

\section{RESULTS}

The histological and histochemical data on these animals are summarized in Tables I to III. A detailed report of the physiological and histochemical data will be published elsewhere (Dubowitz, 1967).

After cross-union of the nerves to the soleus and flexor hallucis longus in the cats and kittens, there were striking histochemical changes in all the flexor hallucis longus muscles (Figs. 1-3). Areas were present composed entirely of type 1 fibres (rich in $\mathrm{NADH}_{2}$ diaphorase, poor in phosphorylase and 6

TABLE I

\begin{tabular}{|c|c|c|c|c|c|c|c|c|c|c|c|}
\hline \multirow{3}{*}{$\begin{array}{l}\text { Animal } \\
\text { No. } \\
\text { (age) } \\
\text { (wt.) } \\
\text { at Operation }\end{array}$} & \multirow{3}{*}{$\begin{array}{l}\text { Time } \\
\text { Post- } \\
\text { operatively } \\
\text { (weeks) } \\
n\end{array}$} & \multirow{3}{*}{ Muscle } & \multirow{3}{*}{ Procedure } & \multicolumn{4}{|c|}{ RESULTS IN ADULT CATS } & \multirow{2}{*}{\multicolumn{4}{|c|}{ Fibre Abnormalities }} \\
\hline & & & & \multirow{2}{*}{$\begin{array}{l}\text { Weight } \\
(g) .\end{array}$} & \multirow[t]{2}{*}{ Histology } & \multirow{2}{*}{$\begin{array}{l}\text { Histo- } \\
\text { chemical } \\
\text { Pattern }\end{array}$} & \multirow{2}{*}{$\begin{array}{l}\text { Fibre } \\
\text { Size }(\mu)\end{array}$} & & & & \\
\hline & & & & & & & & $\begin{array}{l}\text { Giant } \\
\text { Fibres }(\mu)\end{array}$ & Coils & Cores & $\begin{array}{l}\text { Mitochondrial } \\
\text { Abnormality }\end{array}$ \\
\hline $\begin{array}{l}\text { C2 } \\
(1,750 \mathrm{~g} .)\end{array}$ & 33 & $\begin{array}{l}\text { R. SOL } \\
\text { R. FHL } \\
\text { R. FDL } \\
\text { L. SOL } \\
\text { L. FHL } \\
\text { L. FDL }\end{array}$ & $\begin{array}{l}\text { SI } \\
\text { SI } \\
\text { CON } \\
\text { CI } \\
\text { CI } \\
\text { CON }\end{array}$ & $\begin{array}{l}2.43 \\
3.37 \\
0.97 \\
2.06 \\
2.82 \\
1.44\end{array}$ & $\begin{array}{l}\mathbf{N} \\
\mathbf{N} \\
\mathbf{N} \\
\mathbf{N} \\
\mathbf{N} \\
\mathbf{N}\end{array}$ & $\begin{array}{l}\mathbf{N} 2 \\
\mathbf{N} \\
\mathbf{N} \\
\mathbf{N} 2 \\
\mathbf{C} \\
\mathbf{N}\end{array}$ & $\begin{array}{l}45-90 \\
45-90 \\
45-120 \\
45-90 \\
50-90 \\
45-90\end{array}$ & & & & \\
\hline $\begin{array}{l}\text { C3 } \\
(2,600 \text { g. })\end{array}$ & 29 & $\begin{array}{l}\text { R. SOL } \\
\text { R. FHL } \\
\text { R. FDL } \\
\text { L. SOL } \\
\text { L. FHL } \\
\text { L. FDL }\end{array}$ & $\begin{array}{l}\text { CI } \\
\text { CI } \\
\text { CON } \\
\text { DEN } \\
\text { DEN } \\
\text { CON }\end{array}$ & $\begin{array}{l}3.49 \\
1.22 \\
1.90 \\
1.63 \\
\\
2.86 \\
1.09\end{array}$ & $\begin{array}{l}\mathbf{N} \\
\mathbf{N} \\
\mathbf{N} \\
\mathbf{F A} \\
\mathbf{N} \\
\mathbf{N}\end{array}$ & $\begin{array}{l}\mathbf{N} \\
\mathbf{C} \\
\mathbf{N} \\
\mathbf{N} \\
\\
\mathbf{N} \\
\mathbf{N}\end{array}$ & $\begin{array}{l}60-90 \\
30-90 \\
30-75 \\
15-30 \\
30-90 \\
45-90 \\
30-75\end{array}$ & & & & \\
\hline $\begin{array}{l}\text { C5 } \\
(1,750 \mathrm{~g} .)\end{array}$ & 26 & $\begin{array}{l}\text { R. SOL } \\
\text { R. FHL } \\
\text { R. FDL } \\
\text { L. SOL } \\
\text { L. FHL } \\
\text { L. FDL }\end{array}$ & $\begin{array}{l}\text { SI } \\
\text { SI } \\
\text { CON } \\
\text { CI } \\
\text { CI } \\
\text { CON }\end{array}$ & $\begin{array}{l}3.05 \\
3.36 \\
1.58 \\
3.54 \\
2.53 \\
1.83\end{array}$ & $\begin{array}{l}\mathbf{N} \\
\mathbf{N} \\
\mathbf{N} \\
\mathbf{N} \\
\mathbf{N} \\
\mathbf{N}\end{array}$ & $\begin{array}{l}\mathbf{N} \\
\mathbf{N} \\
\mathbf{N} \\
\mathbf{N} \\
\mathbf{C} \\
\mathbf{N}\end{array}$ & $\begin{array}{l}30-60 \\
30-90 \\
30-80 \\
30-60 \\
30-60 \\
45-75\end{array}$ & & $\begin{array}{l}\text { Parasi } \\
\text { Parasi } \\
\text { Parasi } \\
\text { Parasi } \\
\text { Parasi }\end{array}$ & $\begin{array}{l}\text { c cysts } \\
\text { c cysts } \\
\text { c cysts } \\
\text { c cysts } \\
\text { c cysts }\end{array}$ & \\
\hline $\begin{array}{l}\text { C6 } \\
(3,200 \text { g. })\end{array}$ & 27 & $\begin{array}{l}\text { R. SOL } \\
\text { R. FHL } \\
\text { R. FDL } \\
\text { L. SOL } \\
\text { L. FHL } \\
\text { L. FDL }\end{array}$ & $\begin{array}{l}\text { TEN } \\
\text { TEN } \\
\text { CON } \\
\text { DEN } \\
\text { CON } \\
\text { DEN }\end{array}$ & $\begin{array}{l}1 \cdot 38 \\
3 \cdot 99 \\
2 \cdot 16 \\
2 \cdot 15 \\
\\
4 \cdot 54 \\
0 \cdot 40\end{array}$ & $\begin{array}{l}\mathbf{A} \\
\mathbf{N} \\
\mathbf{N} \\
\mathbf{F A} \\
\mathbf{N} \\
\mathbf{A}\end{array}$ & $\begin{array}{l}\mathbf{N} \\
\mathbf{N} \\
\mathbf{N} \\
\mathbf{N} \\
\mathbf{N} \\
\mathbf{N}\end{array}$ & $\begin{array}{l}10-90 \\
30-75 \\
30-60 \\
15-30 \\
45-90 \\
45-60 \\
10-30 \\
30-60\end{array}$ & & $\begin{array}{l}\text { Parasi } \\
\text { Parasi }\end{array}$ & $\begin{array}{l}\text { c cysts } \\
\text { c cysts }\end{array}$ & \\
\hline
\end{tabular}

Key: $\mathbf{N}=$ Normal; $\mathbf{N} 2=$ Normal with variations in fibre reaction; $A=$ atrophy; $\mathbf{F A}=$ Focal atrophy; $C=$ Mixed pattern; $\mathbf{R}=$ regenerating $; \mathbf{C I}=$ Cross-innervation $; \mathbf{S I}=$ Self-innervation $; \mathrm{TEN}=$ Tenotomy $\mathbf{C O N}=\mathbf{C o n t r o l} ; \mathbf{D E N}=\mathbf{D e n e r v a t i o n}$. 
TABLE II

RESULTS IN ADULT CATS AND NEWBORN RABBITS AND KITTENS

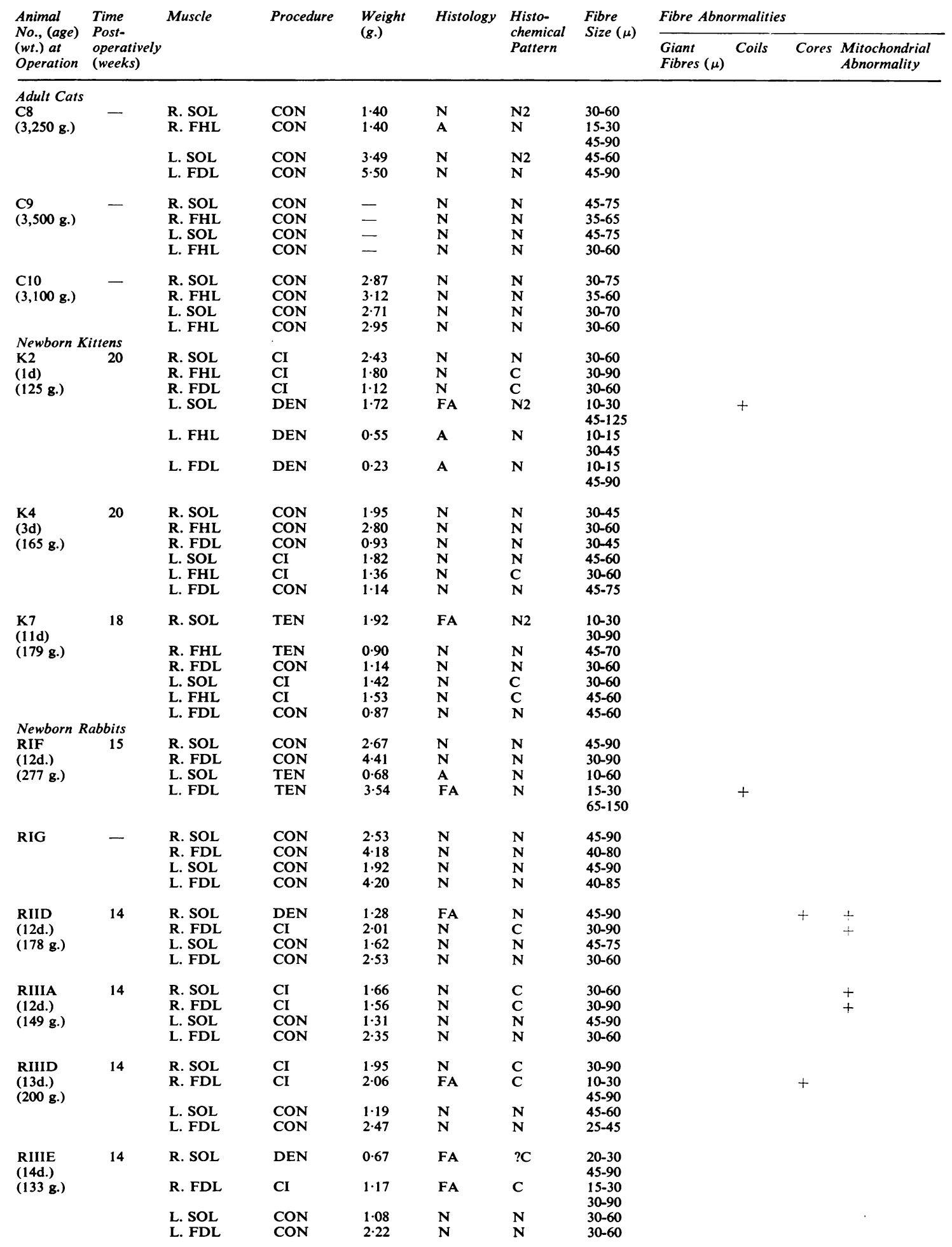


TABLE III

RESULTS IN NEWBORN RABBITS

\begin{tabular}{|c|c|c|c|c|c|c|c|c|c|c|c|}
\hline \multirow{2}{*}{$\begin{array}{l}\text { Animal } \\
\text { No. (age) } \\
\text { (wt.) at } \\
\text { operation }\end{array}$} & \multirow{2}{*}{$\begin{array}{l}\text { Time } \\
\text { Post- } \\
\text { operatively } \\
\text { (weeks) }\end{array}$} & \multirow[t]{2}{*}{ Muscle } & \multirow[t]{2}{*}{ Procedure } & \multirow{2}{*}{$\begin{array}{l}\text { Weight } \\
(\mathrm{g} .)\end{array}$} & \multirow[t]{2}{*}{ Histology } & \multirow{2}{*}{$\begin{array}{l}\text { Histo- } \\
\text { chemical } \\
\text { Pattern }\end{array}$} & \multirow{2}{*}{$\begin{array}{l}\text { Fibre } \\
\text { Size }(\mu)\end{array}$} & \multicolumn{4}{|c|}{ Fibre Abnormalities } \\
\hline & & & & & & & & $\begin{array}{l}\text { Giant } \\
\text { Fibres }(\mu)\end{array}$ & Coils & Cores & $\begin{array}{l}\text { Mitochondrial } \\
\text { Abnormality }\end{array}$ \\
\hline $\begin{array}{l}\text { RVC } \\
(29 d .) \\
(421 \text { g.) }\end{array}$ & 6 & $\begin{array}{l}\text { R. SOL } \\
\text { R. FDL }\end{array}$ & $\begin{array}{l}\text { CI } \\
\text { DEN }\end{array}$ & $\begin{array}{l}0.97 \\
1.81\end{array}$ & & $\stackrel{? C}{C}$ & $\begin{array}{l}45-75 \\
20-45 \\
60-90\end{array}$ & & & & \\
\hline & & $\begin{array}{l}\text { L. SOL } \\
\text { L. FDL }\end{array}$ & $\begin{array}{l}\text { CON } \\
\text { CON }\end{array}$ & $\begin{array}{l}0.65 \\
2.09\end{array}$ & & $\begin{array}{l}\mathbf{N} \\
\mathbf{N}\end{array}$ & $\begin{array}{l}45-60 \\
30-60\end{array}$ & & & & \\
\hline $\begin{array}{l}\text { RVD } \\
\text { (30d.) } \\
(358 \text { g.) }\end{array}$ & 6 & $\begin{array}{l}\text { R. SOL } \\
\text { R. FDL } \\
\text { L. SOL } \\
\text { L. FDL }\end{array}$ & $\begin{array}{l}\text { CI } \\
\text { CI } \\
\text { CON } \\
\text { CON }\end{array}$ & $\begin{array}{l}0.86 \\
1.99 \\
0.92 \\
2.26\end{array}$ & $\begin{array}{l}\mathbf{N} \\
\mathbf{R} \\
\mathbf{N} \\
\mathbf{N}\end{array}$ & $\begin{array}{l}\mathbf{N} \\
\mathbf{C} \\
\mathbf{N} \\
\mathbf{N}\end{array}$ & $\begin{array}{l}30-60 \\
30-90 \\
30-60 \\
45-60\end{array}$ & $90-150$ & & + & + \\
\hline $\begin{array}{l}\text { RVE } \\
(5 d .) \\
(418 \mathrm{~g} .)\end{array}$ & 6 & $\begin{array}{l}\text { R. SOL } \\
\text { R. FDL } \\
\text { L. SOL } \\
\text { L. FDL }\end{array}$ & $\begin{array}{l}\text { CI } \\
\text { CI } \\
\text { CON } \\
\text { CON }\end{array}$ & $\begin{array}{l}1.06 \\
2.25 \\
0.97 \\
2 \cdot 20\end{array}$ & $\begin{array}{l}\mathbf{N} \\
\mathbf{R} \\
\mathbf{N} \\
\mathbf{N}\end{array}$ & $\begin{array}{l}? \mathrm{C} \\
\mathrm{C} \\
\mathbf{N} \\
\mathrm{N}\end{array}$ & $\begin{array}{l}40-60 \\
30-90 \\
30-60 \\
30-60\end{array}$ & $90-120$ & & + & + \\
\hline $\begin{array}{l}\text { RVIA } \\
\text { (5d.) }\end{array}$ & 6 & R. SOL & CI & 0.63 & $\mathbf{A}, \mathbf{R}$ & $\mathbf{N}$ & $\begin{array}{r}5-15 \\
30-60\end{array}$ & & & & + \\
\hline (91g.) & & $\begin{array}{l}\text { R. FDL } \\
\text { L. SOL } \\
\text { L. FDL }\end{array}$ & $\begin{array}{l}\text { CI } \\
\text { CON } \\
\text { CON }\end{array}$ & $\begin{array}{l}0.71 \\
0.45\end{array}$ & $\begin{array}{l}\mathbf{A}, \mathbf{R} \\
\mathbf{N} \\
\mathbf{N}\end{array}$ & $\begin{array}{l}\stackrel{?}{N} \\
\mathbf{N} \\
\mathbf{N}\end{array}$ & $\begin{array}{l}10-70 \\
20-45 \\
15-35\end{array}$ & & & + & + \\
\hline $\begin{array}{l}\text { RVIB } \\
\text { (5d.) } \\
(75 \mathrm{~g} .)\end{array}$ & $\begin{array}{l}6 \\
.\end{array}$ & $\begin{array}{l}\text { R. SOL } \\
\text { R. FDL } \\
\text { L. SOL } \\
\text { L. FDL }\end{array}$ & $\begin{array}{l}\text { CI } \\
\text { CI } \\
\text { CON } \\
\text { CON }\end{array}$ & $\begin{array}{l}0 \cdot 32 \\
0 \cdot 50 \\
0 \cdot 41 \\
0 \cdot 70\end{array}$ & $\begin{array}{l}\mathbf{N}, \mathbf{R} \\
\mathbf{N}, \mathbf{R} \\
\mathbf{N} \\
\mathbf{N}\end{array}$ & $\begin{array}{l}\mathbf{N} \\
? \mathrm{C} \\
\mathbf{N} \\
\mathbf{N}\end{array}$ & $\begin{array}{l}30-65 \\
30-75 \\
30-45 \\
15-40\end{array}$ & 75 & $\begin{array}{l}+ \\
+\end{array}$ & + & + \\
\hline $\begin{array}{l}\text { RVID } \\
\text { (6d.) } \\
(82 \text { g.) }\end{array}$ & 6 & $\begin{array}{l}\text { R. SOL } \\
\text { R. FDL } \\
\text { L. SOL } \\
\text { L. FDL }\end{array}$ & $\begin{array}{l}\text { CI } \\
\text { CI } \\
\text { CON } \\
\text { CON }\end{array}$ & $\begin{array}{l}0 \cdot 44 \\
0 \cdot 60 \\
0 \cdot 37 \\
0 \cdot 70\end{array}$ & $\begin{array}{l}\mathbf{N} \\
\mathbf{R} \\
\mathbf{N} \\
\mathbf{N}\end{array}$ & $\begin{array}{l}\mathbf{N} \\
\mathbf{C} \\
\mathbf{N} \\
\mathbf{N}\end{array}$ & $\begin{array}{l}20-45 \\
15-90 \\
30-45 \\
15-30\end{array}$ & 90 & & + & + \\
\hline $\begin{array}{l}\text { RVIE } \\
\text { (6d.) } \\
(90 \text { g.) }\end{array}$ & 5 & $\begin{array}{l}\text { R. SOL } \\
\text { R. FDL } \\
\text { L. SOL } \\
\text { L. FDL }\end{array}$ & $\begin{array}{l}\text { CI } \\
\text { DEN } \\
\text { CON } \\
\text { CON }\end{array}$ & $\begin{array}{l}0.40 \\
0 \cdot 62 \\
0 \cdot 29 \\
0.82\end{array}$ & $\begin{array}{l}\mathbf{A}, \mathbf{R} \\
\mathbf{R} \\
\mathbf{N} \\
\mathbf{N}\end{array}$ & $\begin{array}{l}? \mathrm{C} \\
\mathrm{C} \\
\mathbf{N} \\
\mathrm{N}\end{array}$ & $\begin{array}{l}30-75 \\
30-75 \\
30-45 \\
20-35\end{array}$ & $75-90$ & + & & + \\
\hline $\begin{array}{l}\text { RVIF } \\
(7 d .) \\
(100 \text { g. })\end{array}$ & 6 & $\begin{array}{l}\text { R. SOL } \\
\text { R. FDL } \\
\text { L. SOL } \\
\text { L. FDL }\end{array}$ & $\begin{array}{l}\text { CI } \\
\text { CI } \\
\text { CON } \\
\text { CON }\end{array}$ & $\begin{array}{l}0.44 \\
0.46 \\
0.41 \\
0.81\end{array}$ & $\begin{array}{l}\mathbf{N} \\
\mathbf{R} \\
\mathbf{N} \\
\mathbf{N}\end{array}$ & $\begin{array}{l}? \mathrm{C} \\
\mathbf{C} \\
\mathbf{N} \\
\mathrm{N}\end{array}$ & $\begin{array}{l}30-45 \\
30-75 \\
25-45 \\
15-35\end{array}$ & 90 & + & $\therefore$ & + \\
\hline $\begin{array}{l}\text { RVIH } \\
\text { (7d.) } \\
(95 \mathrm{~g} .)\end{array}$ & 5 & $\begin{array}{l}\text { R. SOL } \\
\text { R. FDL } \\
\text { L. SOL } \\
\text { L. FDL }\end{array}$ & $\begin{array}{l}\text { DEN } \\
\text { CI } \\
\text { CON } \\
\text { CON }\end{array}$ & $\begin{array}{l}0.40 \\
0.51 \\
0.35 \\
0.75\end{array}$ & $\begin{array}{l}\mathbf{R} \\
\mathbf{R} \\
\mathbf{N} \\
\mathbf{N}\end{array}$ & $\begin{array}{l}\mathbf{N} \\
? \\
\mathbf{N} \\
\mathbf{N}\end{array}$ & $\begin{array}{l}15-75 \\
10-75 \\
30-45 \\
30-45\end{array}$ & $\begin{array}{l}90-120 \\
75-100\end{array}$ & & + & $\dot{I}$ \\
\hline $\begin{array}{l}\text { RVIJ } \\
(7 \mathrm{~d} .) \\
(79 \mathrm{~g} .)\end{array}$ & 5 & $\begin{array}{l}\text { R. SOL } \\
\text { R. FDL } \\
\text { L. SOL } \\
\text { L. FDL }\end{array}$ & $\begin{array}{l}\text { CI } \\
\text { CI } \\
\text { CON } \\
\text { CON }\end{array}$ & $\begin{array}{l}0.25 \\
0.45 \\
0.50 \\
0.90\end{array}$ & $\begin{array}{l}\mathbf{N} \\
\mathbf{R} \\
\mathbf{N} \\
\mathbf{N}\end{array}$ & $\begin{array}{l}? \mathrm{C} \\
\mathrm{C} \\
\mathbf{N} \\
\mathrm{N}\end{array}$ & $\begin{array}{l}15-45 \\
15-75 \\
15-45 \\
15-45\end{array}$ & $75-105$ & + & + & + \\
\hline $\begin{array}{l}\text { RVIIA } \\
\text { (5d.) } \\
\text { (66 g.) }\end{array}$ & 6 & $\begin{array}{l}\text { R. SOL } \\
\text { R. FDL } \\
\text { L. SOL } \\
\text { L. FDL }\end{array}$ & $\begin{array}{l}\text { DEN } \\
\text { DEN } \\
\text { CON } \\
\text { CON }\end{array}$ & $\begin{array}{l}0.26 \\
0.66 \\
0 \cdot 41 \\
0.91\end{array}$ & $\begin{array}{l}\mathbf{F A} \\
\mathbf{R} \\
\mathbf{N} \\
\mathbf{N}\end{array}$ & $\begin{array}{l}\mathbf{N} \\
\mathbf{N} \\
\mathbf{N} \\
\mathbf{N}\end{array}$ & $\begin{array}{l}15-60 \\
15-90 \\
30-60 \\
30-45\end{array}$ & $\begin{array}{l}60-90 \\
75-120\end{array}$ & + & + & \\
\hline $\begin{array}{l}\text { RVIIB } \\
(5 d .) \\
(77 \mathrm{~g} .)\end{array}$ & 6 & $\begin{array}{l}\text { R. SOL } \\
\text { R. FDL } \\
\text { L. SOL } \\
\text { L. FDL }\end{array}$ & $\begin{array}{l}\text { CI } \\
\text { CI } \\
\text { CON } \\
\text { CON }\end{array}$ & $\begin{array}{l}0.42 \\
0.57 \\
0.36 \\
0.65\end{array}$ & $\begin{array}{l}\mathbf{R}, \mathbf{F A} \\
\mathbf{N}, \mathbf{R} \\
\mathbf{N} \\
\mathbf{N}\end{array}$ & $\begin{array}{l}? \mathrm{C} \\
\mathbf{C} \\
\mathbf{N} \\
\mathbf{N}\end{array}$ & $\begin{array}{l}30-60 \\
15-60 \\
15-45 \\
15-40\end{array}$ & $\begin{array}{l}90-120 \\
75-90\end{array}$ & + & & + \\
\hline
\end{tabular}

Key: $\mathbf{N}=$ Normal; $\mathbf{N} 2=$ Normal with variations in fibre reaction; $\mathbf{A}=$ atrophy; $\mathbf{F A}=$ Focal atrophy $\mathbf{C}=$ Mixed pattern; $\mathbf{R}=$ regenerating; $\mathbf{C I}=$ Cross-innervation $; \mathbf{S I}=$ Self-innervation; TEN $=$ Tenotomy; $\mathbf{C O N}=$ Control; $\mathbf{D E N}=$ Denervation. 

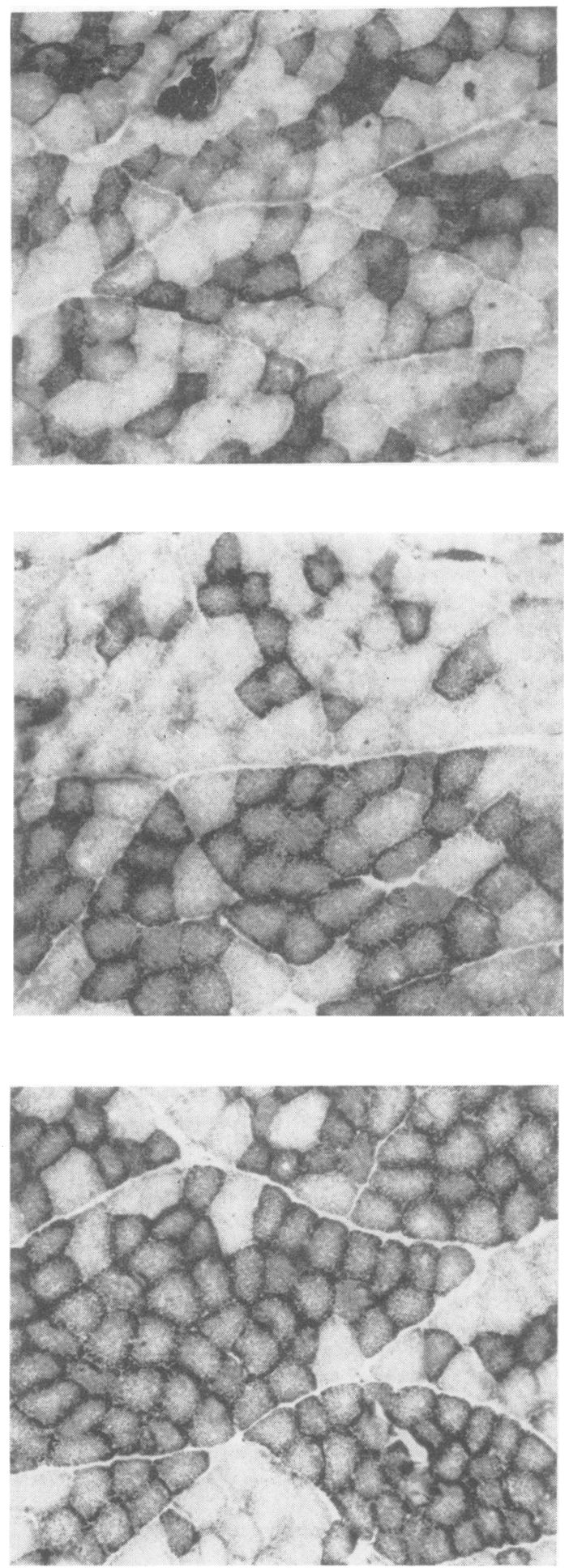

FIG. 1. Cat C5, L. flexor digitorum longus proximal, control. Shows normal flexor digitorum longus pattern consisting of small strongly reacting fibres (type 1), larger weakly reacting fibres (type 2), and intermediate fibres. $\mathrm{NADH}_{2}$ diaphorase. $\times 110$.

FIG. 2. Kitten $K 2, R$. flexor digitorum longus distal, crossinnervated 20 weeks. Part of section composed mainly of weakly reacting type 2 fibres and a small proportion of type 1 fibres. Resembles normal flexor digitorum longus, but type 1 fibres are fewer in number, and no intermediate fibres are present. Remainder of section composed of foci of type 1 fibres, resembling normal soleus. $\mathrm{NADH}_{2}$ diaphorase. $\times 110$.

FIG. 3. Kitten $K 2, R$. flexor digitorum longus distal, cross-innervated 20 weeks. Same muscle as that in Figure 2. Contains three bundles of predominantly type 1 fibres, as in normal soleus. Remaining areas have high proportion of weakly reacting type 2 fibres as in normal flexor digitorum longus. $\mathrm{NADH}_{2}$ diaphorase. $\times 110$. 


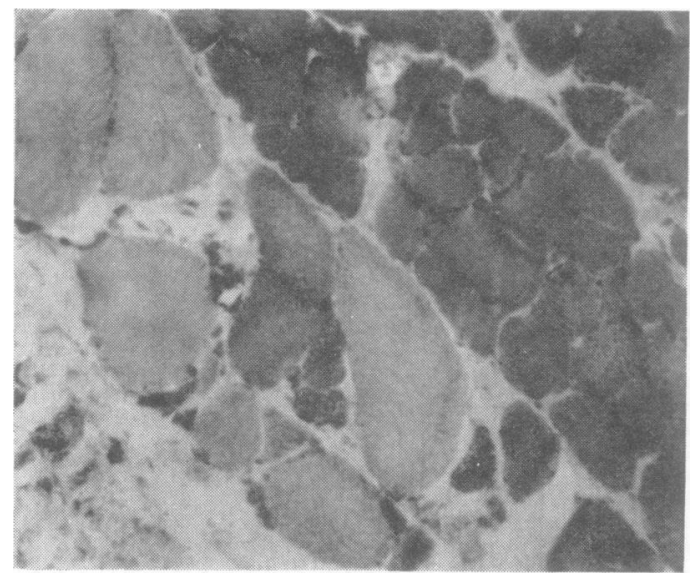

FIG. 4. Kitten K2, L. soleus distal, denervated 20 weeks. Shows a number of bundles of uniformly strong type 1 fibres, as in normal soleus, and one focus of large, weakly reacting type 2 and small type 1 fibres, resembling normal flexor hallucis longus. This area probably resulted from reinnervation from the nerve to the flexor hallucis longus. $\mathrm{NADH}_{2}$ diaphorase. $\times 110$.

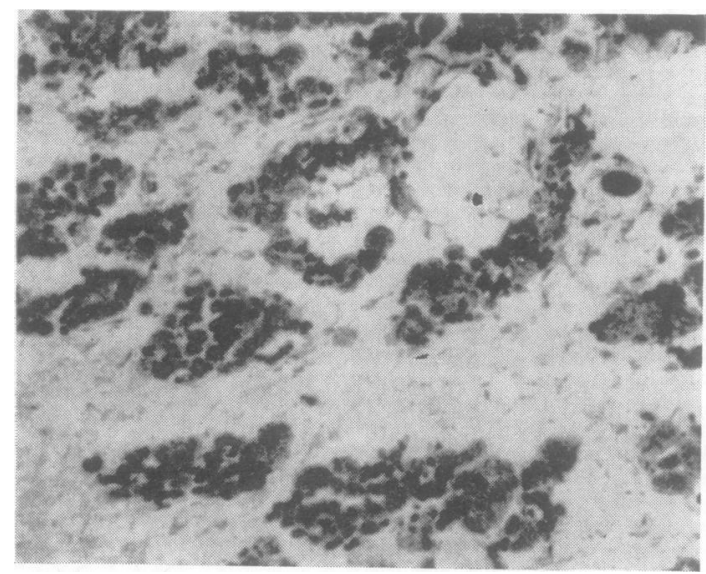

FIG. 5. Kitten K2, L. flexor hallucis longus distal, denervated 20 weeks. Shows universal atrophy of fibres, buto retention of the enzymic pattern of type 1 and type 2 fibres 0 i Note excess of perimysial adipose tissue. $\mathrm{NADH}_{2}$ dia $\mathbb{Q}$ phorase. $\times 110$.

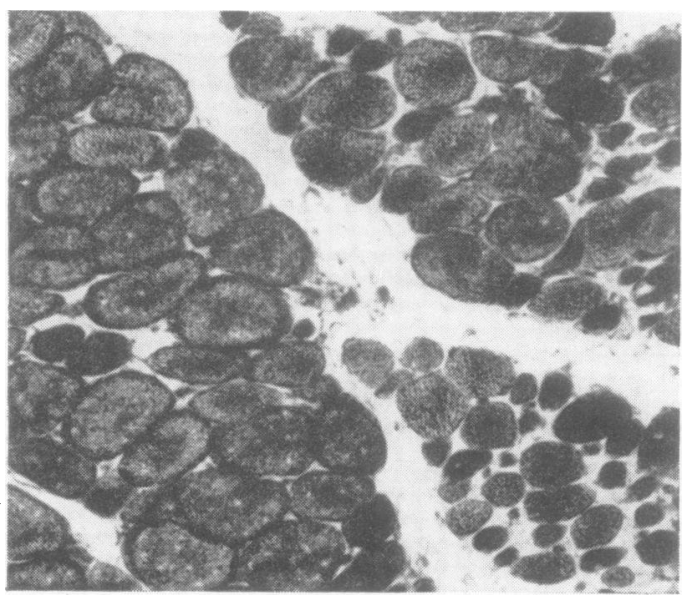

FIG. 6. Cat C6, L. soleus proximal, denervated 27 weeks. Shows area of normal-sized uniformly strongly reacting type 1 fibres, as in normal soleus, and area of atrophic fibres. The normal fibres are presumably re-innervated. $\mathrm{NADH}_{2}$ diaphorase. $\times 110$. 


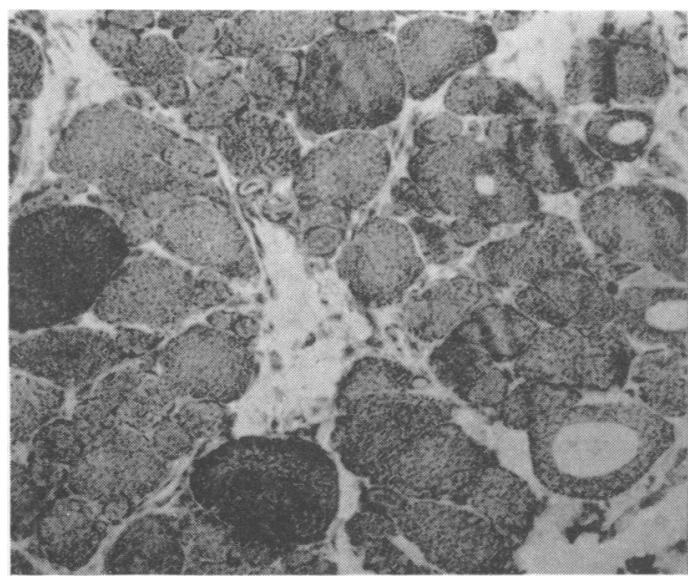

FIG. 7.

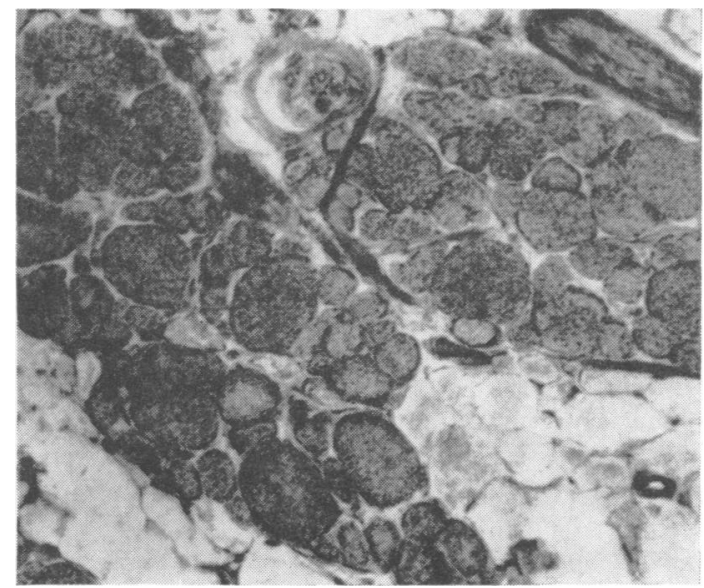

FIG. 8.

FIG. 7. Rabbit VIH, $R$, soleus mid, denervated five weeks. Note variation in fibre size and uniform enzyme activity. Two large coil fibres are present. Four fibres show central vacuolation. Excess of perimysial adipose and connective tissue. $\mathrm{NADH}_{2}$ diaphorase. $\times 110$.

FIG. 8. Rabbit VIH, R. flexor digitorum longus proximal, cross-innervated five weeks. Shows variation in fibre size and in enzymic activity and marked excess of perimysial adipose tissue. Note muscle spindle (top centre) and nerve (top right). $\mathrm{NADH}_{2}$ diaphorase. $\times 140$.

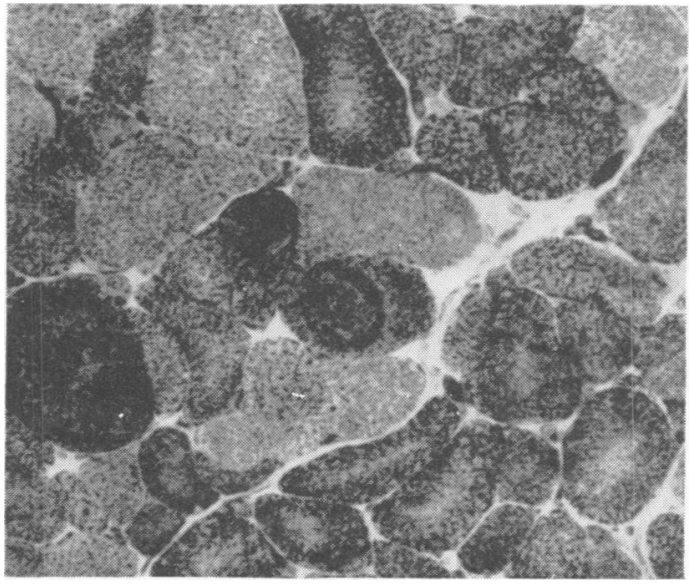

FIG. 9.

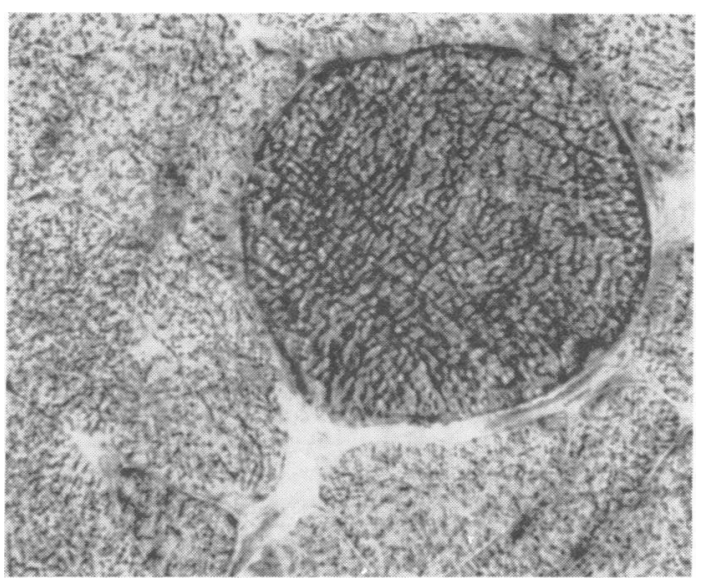

FIG. 10.

FIG. 9. Rabbit VIF, R. flexor digitorum longus proximal, cross-innervated six weeks. Shows marked variation in fibre size, subdivision into strongly reacting type 1 and weakly reacting type 2 fibres, and prominent mitochondria in type 1 fibres. Note central 'core' zones in type 1 fibres and three coil fibres. $\mathrm{NADH}_{2}$ diaphorase. $\times 176$.

FIG. 10. Rabbit VIIB, R. soleus distal, cross-innervated six weeks. Shows giant fibre $(100 \mu)$ with intense enzyme reaction. Remaining fibres uniformly strong as in normal soleus. $\mathrm{NADH}_{2}$ diaphorase. $\times 440$. 
ATPase) and indistinguishable from normal soleus. The remainder of the muscle had the normal checkerboard pattern of flexor hallucis longus with type 1 and 2, although apparently lacking intermediate fibres. The soleus on the other hand showed an equivalent change of pattern in only one instance. No similar changes were observed in any of the selfinnervated or control muscles.

In one denervated soleus muscle (kitten K2) there was one focus of mixed fibres at the periphery (Fig. 4). This consisted of large type 2 fibres and small type 1 fibres, and may have been due to re-innervation by aberrant fibres from the cut nerve to the flexor hallucis longus.

The cross-innervated and self-innervated muscle showed no histological abnormality, and resembled 'quiescent' normal muscle. In contrast, many of the denervated and tenotomized muscles still showed marked histological changes (Figs. 5, 6). Some muscles were still uniformly atrophic (Fig. 5); others showed foci of atrophy amidst areas of normal-sized or enlarged fibres, which were presumably reinnervated (Fig. 6).

In the rabbits the muscles were mostly still in a phase of active regeneration. This was particularly evident in the animals five to six weeks postoperatively. A mixed cross-innervation pattern, similar to that of the cat, was observed in 12 out of 13 cross-innervated flexor digitorum longus muscles, and eight out of 12 soleus muscles. Because of technical difficulty cross-union of both nerves could not be effected in a number of animals. One muscle then was left denervated by resection of the proximal cut nerve. It is of interest that two of the three denervated flexor digitorum longus muscles and one of the four denervated soleus muscles showed a mixed pattern, presumably as a result of re-innervation from the alien proximal nerve stump.

In the regenerating muscles there were striking histological and histochemical changes (Figs. 7-9). Many showed a marked variation in fibre size and shape, and there was excessive perimysial adipose tissue. The pattern was strikingly reminiscent of that seen in muscular dystrophy. These muscle fibres had a marked variation in mitochondrial size and localization as judged by the $\mathrm{NADH}_{2}$ diaphorase reaction and trichrome stain, and many fibres seemed to have prominent mitochondria (Fig. 9), similar to those described by Shy, Gonatas, and Perez (1966). In addition, isolated giant fibres, often more than twice the diameter of remaining fibres were present. These were usually type 1 fibres with a very intense $\mathrm{NADH}_{2}$ diaphorase reaction, but appeared to be structurally normal (Fig. 10).

Morphologically abnormal fibres took various forms. Some resembled ringbinden with a longitud-

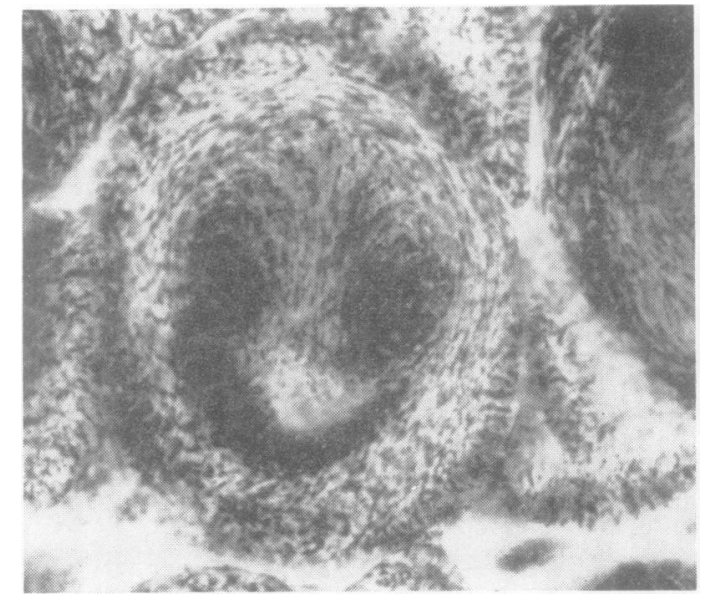

FIG. 11. Rabbit VIIA, R. soleus mid, denervated six weeks. Coil fibre, showing variation in enzyme concentration in fibre. $\mathrm{NADH}_{2}$ diaphorase. $\times 440$.

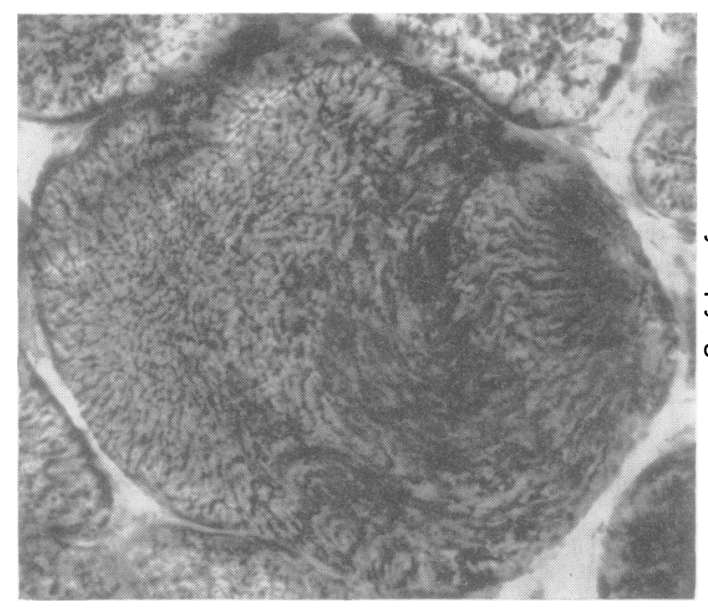

FIG. 12. Rabbit VIIA, R. flexor digitorum longus mid, denervated six weeks. Coil fibre showing different planes in which the coil runs and localization of enzyme activity. $\mathrm{NADH}_{2}$ diaphorase. $\times 440$.

inal coil of muscle, in an otherwise transverse section of the fibre. Others were composed of a snake-like coil of muscle, while some seemed to be subdivided in a random fashion into separate segments (Figs. 11 and 12). In some fibres there was no set pattern but what appeared to be a haphazard disorientation of part of the fibre. Many of these fibres were abnormally large $(120-150 \mu)$.

It was noted that structurally abnormal fibres were frequently aggregated in groups and might be confined to a few adjacent bundles (Fig. 13). These fibres were most common in the rabbits studied five 


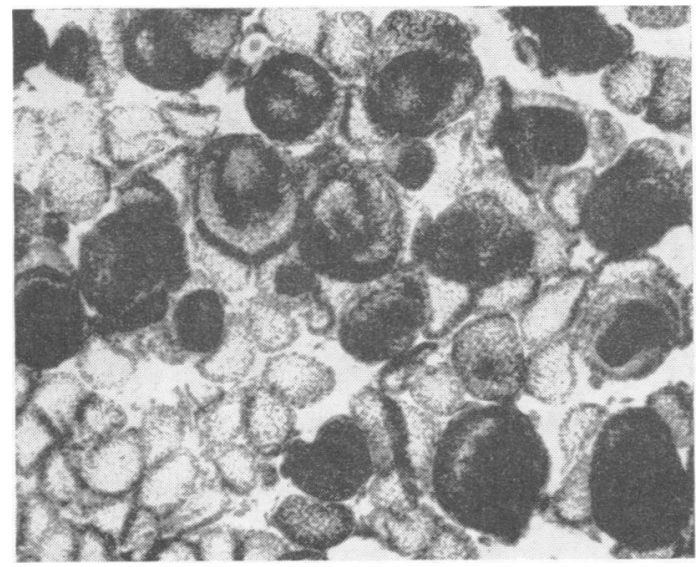

FIG. 13 .

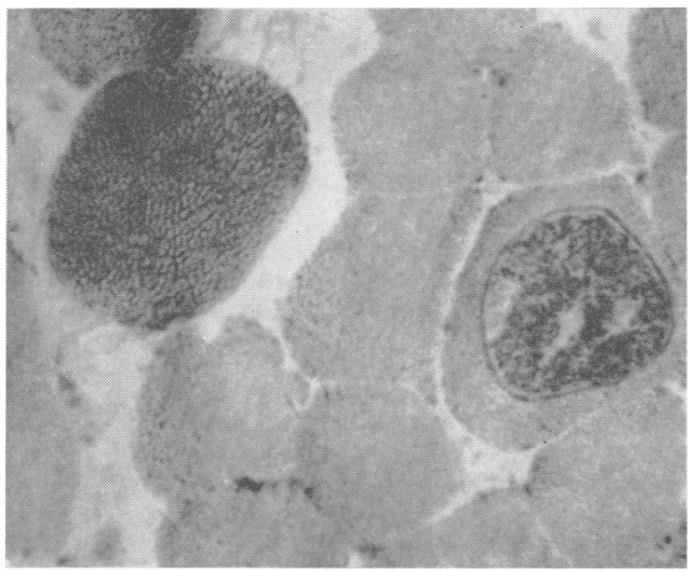

FIG. 14.

FIG. 13. Rabbit VIIA, R. soleus mid, denervated six weeks. Shows large aggregation of coil fibres in one area. $\mathrm{NADH}_{2}$ diaphorase. $\times 275$.

FIG. 14. Cat C5, L. soleus distal, cross-innervated 26 weeks. Shows fibre with parasitic cyst and an isolated giant fibre with intense enzyme activity (cf. Fig. 10). $\mathrm{NADH}_{2}$ diaphorase. $\times 275$.

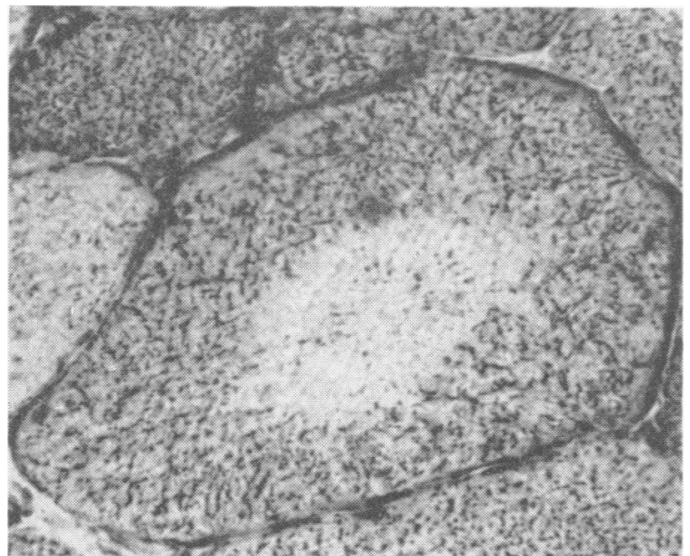

FIG. 15 .

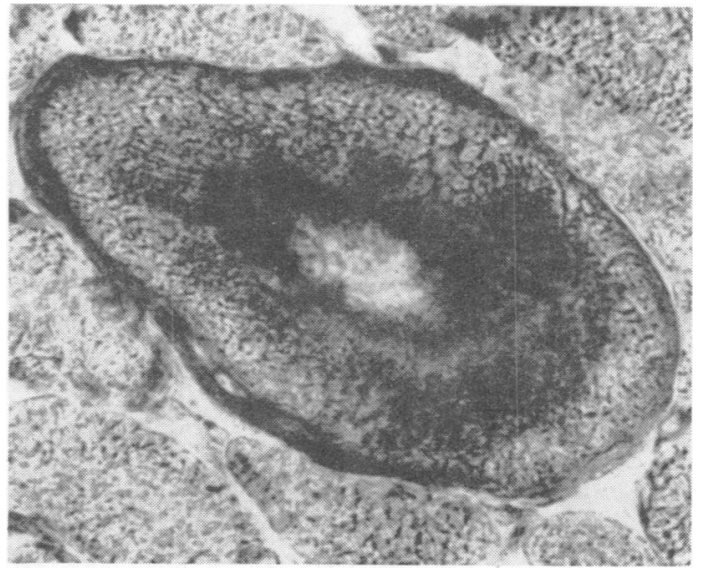

FIG. 16.

FIG. 15. Rabbit VIIA, R. flexor digitorum longus proximal, denervated six weeks. Shows core or targetoid fibre $(150 \times$ $100 \mu)$ with central area devoid of mitochondria and enzymic activity. $\mathrm{NADH}_{2}$ diaphorase. $\times 440$.

FIG. 16. Rabbit VD, R. flexor digitorum longus proximal, cross-innervated six weeks. Target fibre $(90 \times 150 \mu)$ with three zones: inner zone devoid of enzyme activity, middle zone with very strong activity, and outer zone with strong activity. $\mathrm{NADH}_{2}$ diaphorase. $\times 440$. 
or six weeks after operation. The abnormal fibres noted in two adult cats (C5 and C6) were atypical and occurred in re-innervated, tenotomized, and control muscle (Fig. 14). They consisted of a uniform granular central area strong in $\mathbf{N A D H}_{2}$ diaphorase and weak in phosphorylase, surrounded by a narrow rim of apparently normal muscle. They may well be parasitic cysts. Typical coil fibres occurred in the soleus of kitten $\mathrm{K} 2$.

Core fibres took various forms. In the majority there were only two zones, an inner one devoid of enzyme activity and an outer, apparently normal, zone (Fig. 15). These were very similar to the core fibres of central core disease (Dubowitz and Pearse, $1960)$ or the targetoid fibres of Engel (1962). Other fibres had three or more zones (Fig. 16), as in the target fibres of Engel (1962). Some of these target fibres were normal in size, but others were large.

An additional abnormality, thought initially to be an artifact, may be of some significance. Fibres were observed, which appeared to have a round hole in the centre, devoid of any structure and resembling the artifact produced by slow freezing of muscle (Fig. 7). However, these fibres were isolated, surrounded by fibres without similar change, and often in close proximity to a structurally abnormal fibre. They may thus represent vacuolation of the fibre.

\section{DISCUSSION}

Gütmann, Guttmann, Medawar, and Young (1942) studied the rate of regeneration of the motor nerve in the rabbit after section and primary suture. In the distal stump of the peroneal nerve they found an average rate of growth of $2.0 \mathrm{~mm}$. per day, although the fastest axons might regenerate up to $3.5 \mathrm{~mm}$. per day. There was a latent period of about seven days for crossing the gap between the cut ends, and a further latency of about 30 days before restoration of function of the muscle. In young animals (less than 1 month) the rate of growth was about the same $(2.5 \mathrm{~mm}$. per day), but the total latent period was very much shorter (about 16 days).

Since in our experiments the nerves were sectioned within a few millimetres of their distal ends, regeneration must have proceeded to the point of reinnervation of the muscle within six weeks in the adult and probably less than four weeks in the young animals.

During re-innervation, muscle looks grossly 'pathological'. There is a marked variation in fibre size, with random distribution, and a tendency to rounding of fibres and loss of their normal shape in transverse section. The picture bears a very striking resemblance to that of muscular dystrophy.
Structurally abnormal fibres are common and probably represent disordered morphogenesis. These changes appear to be confined to the early phases of re-innervation, after which the muscle becomes histologically normal. The structurally abnormal fibres might either resolve into a normal pattern or else be removed. However, no evidence of phagocytosis or degeneration of these fibres was seen.

The work of Sunderland and Ray (1950) is of interest in this context. In the adult opossum they made a detailed study of changes in muscle after denervation by removing the whole length of the median and ulnar nerves. Muscles were studied from nine to $\mathbf{4 8 5}$ days after denervation. Any animals showing signs of re-innervation were excluded. No structurally abnormal or enlarged fibres were found.

It seems reasonable to assume that the hypertrophied fibres and the coil fibres, observed by Hogenhuis and Engel (1965) in the guinea-pig seven weeks after denervation, may be features of reinnervation and not denervation. The target fibres of Engel (1961) in human neurogenic atrophies may have a similar significance.

In addition to the influence of the nervous system on the contractile properties of muscle (Buller et al. 1960b), we have also demonstrated a markect influence on the metabolic activity, as judged by the transformation in enzymic pattern of the muscle after cross-innervation. At birth all skeletal muscles tends to be slow (Buller, Eccles, and Eccles, 1960a) which correlates with the histochemical observations that in animals skeletal muscle is initially of one type (Dubowitz, 1965a). The slow fibres initially become faster and then slow down again to adult level, while the fast fibres become progressively faster until the adult level is reached (Buller et al., 1960a). In young kittens, transection and deafferentation of the spinal cord has little influence on the normal development of the fast muscle. However, the slow muscle after this procedure becomes fast. Buller et al. (1960a) concluded that the neural influence on muscle was predominantly on the slow fibre.

These results can now be extended to explain various other phenomena. The three phases that human muscle passes through during foetal life (Dubowitz, 1965b) are probably under control of the nervous system. The initial undifferentiated phase may reflect a period before innervation; the second phase, of predominantly type 2 fibres, a period when many of the corresponding nerves have innervated the muscle; the third a transition to the adult form of approximately equal proportions of the two types of muscle fibre as a result of altered innervation.

It is not yet quite clear what type the initial muscle fibres in the human foetus correspond to. In the developing chick Cosmos (1966) has shown that all 
the muscle fibres of the pectoralis and gastrocnemius are initially type 1 and correspond to red muscle. In the developing cat and rabbit the soleus at birth has a histochemical pattern much like the mature adult soleus. Similarly, the flexor hallucis longus and flexor digitorum longus also have a fairly uniform pattern initially and only develop their checkerboard pattern in the second week (Dubowitz, unpublished observations).

In longstanding neurogenic atrophies in humans, the classical picture is one of uniform atrophy of large numbers of fibres interspersed with isolated, or groups of, normal-sized or enlarged fibres. Histochemical study revealed these apparently normal or hypertrophied fibres to be surprisingly uniform in enzyme type (Dubowitz and Pearse, 1961; Fenichel and Engel, 1963; Dubowitz, 1966). Moreover, they might correspond to either type 1 or type 2 fibres or a mixture (Dubowitz, 1966). This was difficult to explain if they were normal fibres which had escaped the neurogenic process, since they should then have had the mixed enzymic pattern of normal muscle. The present studies suggest that hypertrophied fibres are a feature of re-innervated muscle and the uniform enzymic pattern in the human disease presumably represents re-innervation by nerve fibrils normally innervating only one enzymic type of muscle fibre, corresponding either to type 1 or type 2 . The tendency of the re-innervated fibres to occur in groups may be due to branching of the regenerating nerves once they reach the muscle.

The importance of the central nervous system in muscle physiology, metabolism, and pathology raises a number of questions in relation to our understanding of muscle disease. Are there really genetically determined 'primary' diseases of muscle or is not muscular dystrophy perhaps the result of an aberration of the normal controlling influence of the nervous system on muscle? By traditional concept, neurogenic atrophies are the result of a structural degeneration or mechanical interruption of the lower motor neurone. We may in addition have to think in terms of the effects of a 'denervation' or abnormal 'innervation' resulting from a change in the chemical or physiological control of the muscle fibres.

\section{SUMMARY}

The effects of crossed-innervation, self-innervation, denervation, and tenotomy on the histochemical and morphological features of fast and slow muscle have been studied in newborn kittens and rabbits and in adult cats.

Crossed-innervation produced a transformation in the enzymic pattern of the muscle. This was more consistent in the fast flexor hallucis longus or flexor digitorum longus following innervation by the soleus nerve, than vice versa.

During the phase of re-innervation of the muscle structural abnormalities were common. These included core fibres (targetoid and target), coil fibres, occasional ringbinden, giant fibres, and vacuolation of fibres. There was also striking variation in fibre size resembling primary myopathy.

The central nervous system has an important influence not only on the contractile properties of muscle but also on the structural and metabolic character.

I wish to thank Mrs. Joan Steward for her excellent technical assistance; Mr. Donald Newman for his enthusiastic help during the summer; Miss B. Turkington for photographic assistance and Dr. Ethel Cosmos for much helpful advice and discussion. This work was supported by grants from the Muscular Dystrophy Associations of America.

\section{REFERENCES}

Adams, R. D., Denny-Brown, D., and Pearson, C. M. (1962). Diseases of Muscle. 2nd. ed. Harper, New York.

Bergstrand, C. G. (1938). Zur morphologie der quergestreiften Ringbinden. $Z$. mikr-anat. Forsch., 44, 45-55.

Buller, A. J., Eccles, J. C., and Eccles, R. M. (1960a). Differentiation of fast and slow muscles in the cat hind limb. J. Physiol. (Lond.), 150, 399-416.

,$- \quad-, 1$ (1960b). Interactions between motoneurones and muscles in respect of the characteristic speeds of their responses. Ibid., 150, 417-439.

Cosmos, E. (1966). Enzymatic activity of differentiating muscle fibers. 1. Development of phosphorylase in muscles of the domestic fowl. Develop. Biol., 13, 163-181.

Dubowitz, V. (1965a). Enzyme histochemistry of skeletal muscle. Part I. Developing animal muscle. J. Neurol. Neurosurg. Psychiat., 28, 516-519.

(1965b). Enzyme histochemistry of skeletal muscle. Part II. Developing human muscle. Ibid., 28, 519-524.

- (1966). Enzyme histochemistry of skeletal muscle. Part III. Neurogenic muscular atrophies. Ibid., 29, 23-28.

(1967). In preparation.

- and Pearse, A. G. E. (1960). Oxidative enzymes and phosphorylase in central-core disease of muscle. Lancet, 2, 23-24.

- - - (1961). Enzymic activity of normal and diseased human muscle: a histochemical study. J. Path. Bact., 81, 365-378. (1964). Histochemical aspects of muscle disease. In Disorders of Voluntary Muscle, edited by J. N. Walton, pp. 194-219. Churchill, London.

Engel, W. K. (1961). Muscle target fibres, a newly recognized sign of denervation. Nature (Lond.), 191, 389-390.

denervation. Nature (Lond.), The essentiality of histo- and cytochemical studies of
(1962). The skeletal muscle in the investigation of neuromuscular disease. Neurology (Minneap.), 12, 778-794.

- - and Cunningham, G. C. (1963). Rapid examination of muscle tissue. An improved trichrome method for fresh-frozen biopsy sections. Ibid., 13, 919-923.

- Foster, J. B., Hughes, B. P., Huxley, H. E., and Mahler, R. (1961). Central core disease-An investigation of a rare muscle cell abnormality. Brain, 84, 167-185.

Eränkö, O., and Palkama, A. (1961). Improved localization of phosphorylase by the use of polyvinyl pyrrolidone and high substrate concentration. J. Histochem. Cytochem., 9, 585.

Fenichel, G. M., and Engel, W. K. (1963). Histochemistry of muscle in infantile spinal muscular atrophy. Neurology (Minneap.), 13, 1059-1066.

Günther, P. G. (1952). Einfache und komplizierte Ringbindenformen in der quergestreiften Skeletmuskulatur. Virchows. Arch. path. Anat., 322, 214-220. 
Gütmann, E., Guttmann, L., Medawar, P. B., and Young, J. Z. (1942). The rate of regeneration of nerve. J. exp. Biol., 19, 14-44.

Heidenhain, M. (1918). Uber progressive Veränderungen der Muskulatur bei Myotonia atrophica. Beitr. path. Anat., 64, 198-225.

Hogenhuis, L. A. H., and Engel, W. K. (1965). Histochemistry and cytochemistry of experimentally denervated guinea pig muscle. I. Histochemistry. Acta anat. (Basel), 60, 39-65.

Padykula, H. A., and Herman, E. (1955). The specificity of the histochemical method for adenosine triphosphatase. J. Histochem. Cytochem., 3, 170-195.

Perry, R. E., Smith, A. G., and Wrenn, R. N. (1956). Ringbinding of skeletal muscle. Arch. Path., 61, 450-455.

Sawyer, D., Sie, H., and Fishman, W. H. (1965). A technique for preparing permanent histochemical preparation of liver phosphorylase. J. Histochem. Cytockem., 13, 605-607.

Scarpelli, D. G., Hess, R., and Pearse, A. G. E. (1958). The cytochemical localization of oxidative enzymes. I. Diphospho- pyridine nucleotide diaphorase and triphosphopyridine nucleotide diaphorase. J. biophys. biochem. Cytol., 4, 747-752.

Shy, G. M., Gonatas, N. K., and Perez, M. (1966). Two childhood myopathies with abnormal mitochondria. I. Megaconial myopathy II. Pleoconial myopathy. Brain, 89, 133-158.

Sunderland, S., and Ray, L. J. (1950). Denervation changes in mammalian striated muscle. J. Neurol. Neurosurg. Psychiat., 13, 159-177.

Takeuchi, T., and Kuriaki, H. (1955). Histochemical detection of phosphorylase in animal tissues. J. Histochem. Cytochem., 3, 153-160.

Wohlfart. G. (1932). Quergestreifte Ringbinden in normalen Augenmuskeln. Z. mikr.-anat. Forsch., 29, 592-604.

- (1951). Dystrophia myotonica and myotonia congenita: histopathologic studies with special reference to changes in the muscles. J. Neuropath. exp. Neurol., 10, 109-124. 